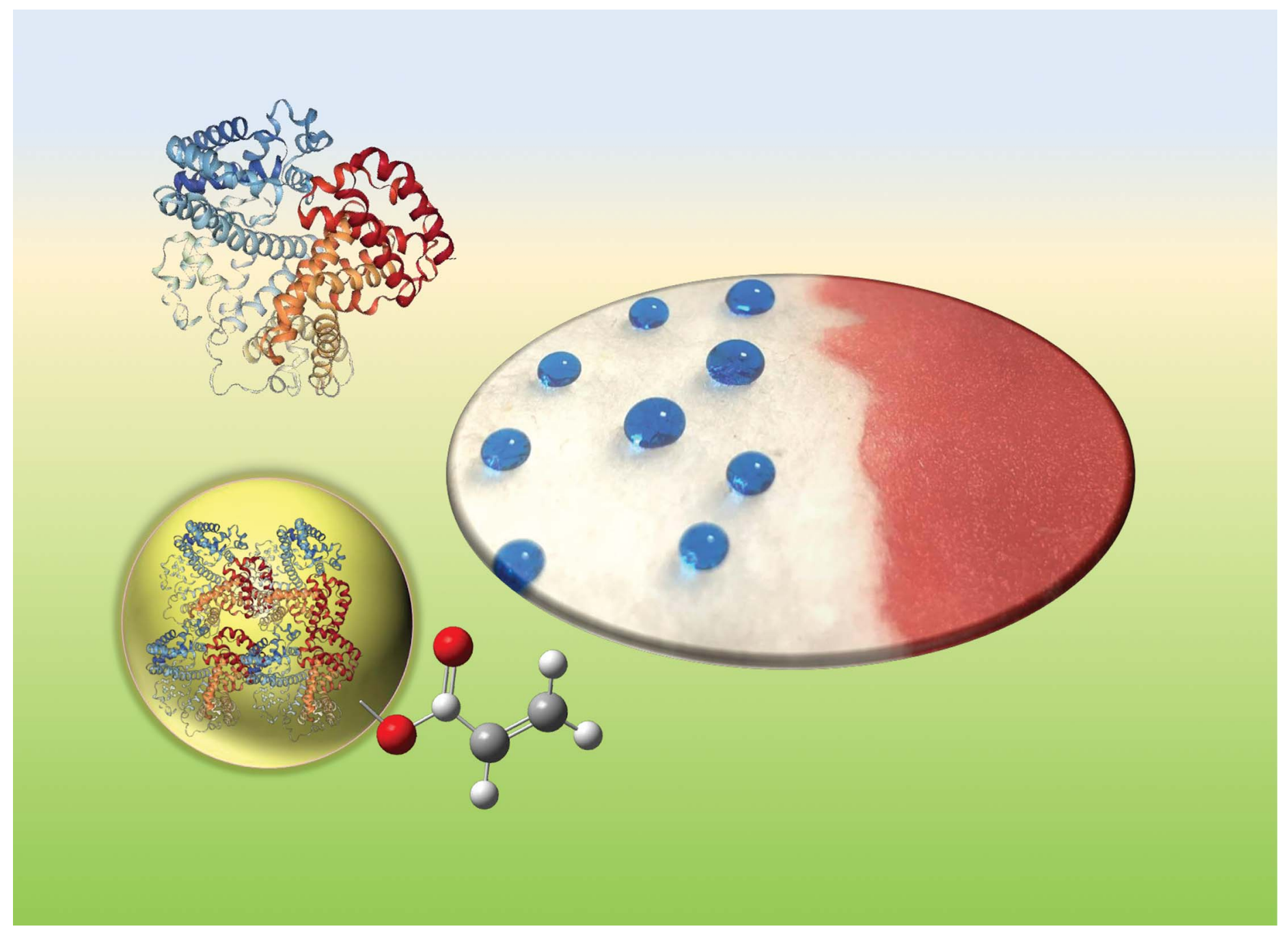

Showcasing research from Dr Manna's Bio-inspired Polymeric Materials laboratory, Department of Chemistry, Indian Institute of Technology-Guwahati, Kamrup, Assam, India.

Chemically reactive protein nanoparticles for synthesis of a durable and deformable superhydrophobic material

This research presents a facile and scalable process for developing a nature-inspired water-repellent interface on an eco-friendly and naturally abundant fibrous substrate, where naturally abundant and biodegradable bovine serum albumin protein-derived chemically reactive nanoparticles are strategically associated with cotton fibers. This protein-based artificial superhydrophobic interface can endure severe repetitive physical manipulations, abrasions, and prolonged chemical exposure, and can also be used for selective absorption of oil spills, irrespective of the density and viscosity of the contaminating oil. Such a protein-derived biomimicking interface is useful for developing various functional materials.

\section{As featured in:}

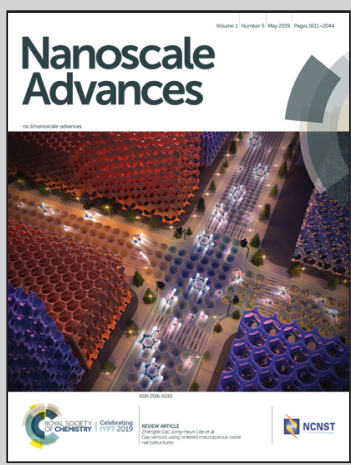

See Uttam Manna et al., Nanoscale Adv., 2019, 1, 1746. 
Check for updates

Cite this: Nanoscale Adv., 2019, 1, 1746

\title{
Chemically reactive protein nanoparticles for synthesis of a durable and deformable superhydrophobic material $\dagger$
}

\author{
Arpita Shome, ${ }^{a}$ Adil Majeed Rather ${ }^{a}$ and Uttam Manna (iD *ab
}

The past few decades have witnessed significant development in the field of artificially biomimicking extremely water repellent interfaces, developed mostly through tedious synthetic processes using synthetic/non-biodegradable polymers and fluorinated derivatives rendering health and environment related hazards. Only a few approaches furnish superhydrophobic materials that can withstand different harsh environments. Here, in this current design, naturally abundant and biodegradable bovine serum albumin (BSA) protein nanoparticles and cotton fibers are rationally selected for environment-friendly green synthesis of a highly sustainable and deformable artificial superhydrophobic material through strategic association of facile and rapid Michael addition reactions between amine and acrylate moieties under ambient conditions without the aid of any catalyst. This protein based nature-inspired interface can endure severe repetitive physical manipulations, abrasions and prolonged (30 days) chemical exposure i.e. extremes of $\mathrm{pH}$, artificial sea water, river water and surfactant contaminated water. This highly durable and compressible superhydrophobic material was successfully exploited for efficient (above $2000 \mathrm{wt} \%$ ), selective and repetitive removal of contaminating oils from aqueous phases under harsh chemical conditions. Such a durable biomimicking interface derived directly from serum protein following a facile synthetic approach would be useful for developing various other functional materials.

Received 16th January 2019

Accepted 6th March 2019

DOI: 10.1039/c9na00031c

rsc.li/nanoscale-advances

\section{Introduction}

Extremely water repellent interfaces are artificially synthesized by mimicking the essential features and chemistry present in the 'lotus-leaf', ${ }^{1-4}$ and have immense potential for resolving various health and environment related issues, including controlled and triggered drug delivery, tissue engineering, antibacterial coatings, eco-friendly remediation of oil spills etc. ${ }^{5-15}$ However, mostly synthetic polymers, inorganic components and fluorinated compounds are used to develop these artificial superhydrophobic materials, ${ }^{1-18}$ which are known to have adverse effects on both the environment and health. ${ }^{19}$ The combination of appropriate hierarchical (micro/nano) structures and essential low surface energy coatings is the essential criterion for developing artificial superhydrophobic surfaces..$^{20,21}$ Most of the hierarchical structures are inherently fragile to various mechanical forces including physical

\footnotetext{
${ }^{a}$ Department of Chemistry, Indian Institute of Technology-Guwahati, Kamrup, Assam 781039, India. E-mail: umanna@iitg.ac.in

${ }^{b}$ Centre for Nanotechnology, Indian Institute of Technology-Guwahati, Kamrup, Assam 781039, India

$\dagger$ Electronic supplementary information (ESI) available: Detailed description of the material and methods for the synthesis of the material and related demonstrations, different physical and chemical durability of the synthesized material and demonstration of light and heavy oil separation and collection. See DOI: $10.1039 / \mathrm{c} 9$ na00031c
}

abrasion, stretching, compression etc., which results in the loss of superhydrophobicity. ${ }^{22-26}$ Furthermore, appropriate low surface energy coatings are generally optimized in artificially fabricated superhydrophobic surfaces by associating with weak chemical interactions, including metal-thiol interaction, ${ }^{27,28}$ metal-ion interaction ${ }^{29,30}$ and silane chemistry, ${ }^{31,32}$ which are known to be labile ${ }^{33}$ and unsustainable in practically relevant harsh aqueous chemical conditions. Hence, many of the reported superhydrophobic interfaces are inappropriate for performing in practically relevant severe and diverse conditions. As a consequence, the demonstrations of oil/water separation under practically relevant harsh conditions are rare in the literature. ${ }^{34-36}$ In the recent past, few early attempts were made to develop artificial superhydrophobicity using naturally existing and biodegradable components including cellulose, chitosan etc. However, the durability of these synthesized materials under challenging conditions is a major concern, where superhydrophobicity is compromised after washing the chitosan derived superhydrophobic interface. ${ }^{37,38}$ Thus, the further design of a durable superhydrophobic substrate from naturally abundant ingredients is essential for sustainable and safe application of this nature-inspired wettability in practically relevant severe scenarios. Here, in this current design, a naturally existing biodegradable biomacromolecule, bovine serum albumin (BSA) protein, ${ }^{39,40}$ has been strategically and unprecedentedly exploited for the facile and scalable synthesis of 
a durable and highly deformable superhydrophobic coating for eco-friendly remediation of various oil-spills in real world scenarios.

In the recent past, BSA protein was successfully deposited on various nanomaterials for surfactant-free stabilization of colloidal dispersion, improving therapeutic properties, synergistic therapy of tumors, exfoliation of transition metal dichalcogenides, photothermal therapy of cancer cells etc. ${ }^{41-47}$ Furthermore, nanoparticles of BSA have been widely exploited for therapeutic applications. ${ }^{4-47}$ This economic and naturally abundant serum protein was successfully exploited for the synthesis of a covalently cross-linked and chemically reactive coating (referred to as the BSA coating) on the fibrous water absorbent cotton for developing a highly tolerant superhydrophobic material. In this current approach, the Michael addition reaction that allowed a catalyst free and rapid chemical reaction, between amine and acrylate groups under ambient conditions, ${ }^{48-50}$ is used for covalently integrating the BSA protein nanoparticles, and the residual acrylate groups in the BSA-coating provided a facile basis for post-covalent modification with primary amine containing small molecules. This simple process eventually yielded durable superhydrophobicity, and the synthesized material is efficient in sustaining repetitive physical deformations, prolonged (30 days) exposure to UV radiation and various other practically relevant chemically severe challenges without compromising the embedded extreme water repellency.

\section{Results and discussion}

\section{Synthesis of chemically reactive BSA protein nanoparticles}

In the past, the nanoparticles of serum proteins were mostly synthesized following the standard desolvation technique, where the protein nanoparticles are desolvated by the addition of ethanol followed by covalent cross-linking with glutaraldehyde molecules. ${ }^{46,47}$ In our current approach, the BSA protein nanoparticles $\left(10 \mathrm{mg} \mathrm{mL}^{-1}\right)$ that were synthesized by adding ethanol were strategically exposed to dipentaerythritol pentaacrylate (5Acl), instead of glutaraldehyde as shown in Fig. 1A, to develop the chemically reactive and covalently cross-linked protein nanoparticles. The amine groups in the BSA protein readily react with acrylate groups through a Michael addition reaction under ambient conditions, ${ }^{48-50}$ without the aid of any catalyst as shown in Fig. 1B, and this strategic cross-linking of BSA nanoparticles using multifunctional small molecules (5Acl) provided residual chemical reactivity to the granular BSA nanoparticles (average diameter: $487 \mathrm{~nm} \pm 16.85$ ). The appearance of the IR peaks due to (a) the asymmetric $\mathrm{C}-\mathrm{H}$ stretching (at $1410 \mathrm{~cm}^{-1}$ ) of $\beta$ carbon of the vinyl group and (b) stretching of the carbonyl group (at $1736 \mathrm{~cm}^{-1}$ ) together revealed the presence of unreacted acrylate groups in the BSA nanoparticles as shown in Fig. 1D. ${ }^{49}$ Hence, these residual acrylate groups provided further scope for post-covalent modification with primary amine containing small molecule i.e. ODA. The treatment of these reactive BSA nanoparticles with octadecylamine (ODA) resulted in the change of zeta potential from $-10.9 \mathrm{mV}$ to $-5.03 \mathrm{mV}$ at $\mathrm{pH}$ 7. The long
A
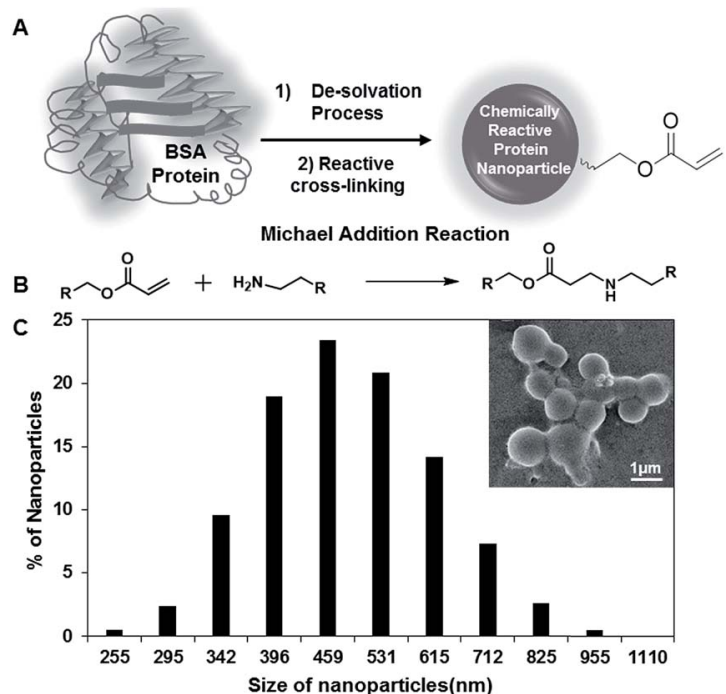

D

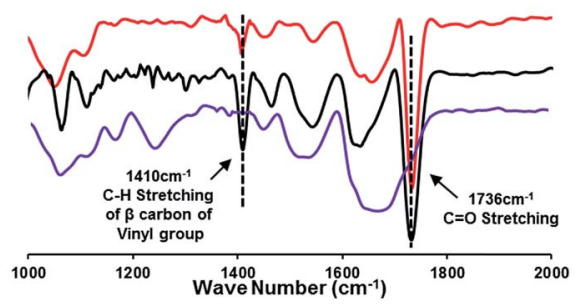

Fig. 1 (A) Schematic illustrating the formation of the chemically 'reactive' bovine serum albumin nanocomplex by desolvating BSA protein from aqueous media using ethanol followed by covalent cross-linking through a Michael addition reaction (B). (C) DLS data showing the size of the 'reactive' BSA nanocomplex. Inset is the FESEM image of the covalently cross-linked BSA nanocomplex. (D) FTIR spectra of a BSA nanoparticle (violet) after covalent cross-linking (black) with $5 \mathrm{Acl}$ molecules, and after post-modification with octadecylamine (red). The peaks at $1736 \mathrm{~cm}^{-1}$ and $1410 \mathrm{~cm}^{-1}$ correspond to the carbonyl stretching and symmetric deformation of the $\mathrm{C}-\mathrm{H}$ bond for the $\beta$ carbon of the vinyl group, respectively.

hydrocarbon tail of attached ODA molecules is likely to screen the surface charge of the BSA protein nanoparticles. Furthermore, the significant depletion in the IR peak intensity for the asymmetric C-H stretching (at $1410 \mathrm{~cm}^{-1}$ ) of $\beta$ carbon of the vinyl group with respect to the carbonyl stretching strongly suggested the covalent reaction between the chemically reactive protein nanoparticles and ODA through a Michael addition reaction under ambient conditions. The stretching vibration of carbonyl groups provided internal reference for this Michael addition reaction as the carbonyl moiety remained unaltered during the course of this mutual reaction between acrylate and amine groups; however, the vinyl moiety of the acrylate groups is compromised. This FTIR characterization and zeta potential analysis unambiguously revealed the unprecedented synthesis of primary amine reactive BSA nanoparticles.

\section{Development of protein-derived superhydrophobicity}

This simple synthetic approach of catalyst-free covalently cross-linked and chemically reactive protein nanoparticles 
was extended for developing nature-inspired durable superhydrophobicity. In the presence of naturally abundant fibrous medical cotton, the same desolvation process was repeated for directly depositing the BSA nanoparticles on the selected fibrous substrate. Upon addition of ethanol, (a) BSA nanoparticles were immediately deposited on the fibrous substrate, and (b) upon treatment with 5Acl molecules, a covalently cross-linked uniform coating of BSA nanocomplexes on the selected fibrous substrate was formed as characterized by FESEM imaging (Fig. 2A and B). The granular domains randomly aggregated and provided the essential hierarchical topography for achieving extreme water repellency. The uncoated. i.e., pristine cotton otherwise has a smooth and uniform surface as shown in Fig. S1G. $\uparrow$ On the other hand, the 5Acl treated BSA coating that consists of residual acrylate groups provided a facile basis for covalent modulation of essential chemistry in the deposited BSA coating on the fibrous substrate through appropriate primary amine containing small molecules. Octadecylamine (ODA) molecules that consist of a long hydrocarbon tail $\left(-\mathrm{C}_{18} \mathrm{H}_{39} \mathrm{~N}\right)$ and primary amine group were strategically and covalently integrated with this hierarchically featured and chemically reactive BSA coating through a 1,4-conjugate addition reaction for adopting the appropriate chemistry to confer extreme water repellency. An optimum concentration of BSA, i.e., 10 $\mathrm{mg} \mathrm{mL}^{-1}$ is required to ensure uniform formation and deposition of the nanoparticles. Concentrations less than the optimized value, i.e., $3 \mathrm{mg} \mathrm{mL}^{-1}, 5 \mathrm{mg} \mathrm{mL}^{-1}$ and $7 \mathrm{mg} \mathrm{mL}^{-1}$ failed to provide a superhydrophobic interface as the percentage of nanoparticle deposition is exceedingly reduced. Only $1.56 \mathrm{wt} \%, 3.42 \mathrm{wt} \%$, and $4.31 \mathrm{wt} \%$ of BSA nanoparticles were deposited for $3 \mathrm{mg} \mathrm{mL} L^{-1}, 5 \mathrm{mg} \mathrm{mL}^{-1}$ and $7 \mathrm{mg} \mathrm{mL}^{-1}$ concentrations of BSA solution respectively, whereas 7\% deposition was calculated for $10 \mathrm{mg} \mathrm{mL}{ }^{-1}$ concentration of BSA. After post-chemical modification with octadecylamine (ODA), the BSA derived coating that was prepared with a lower concentration $\left(3 \mathrm{mg} \mathrm{mL}{ }^{-1}\right.$ ) of BSA is hydrophilic (water contact angle (WCA) of $80^{\circ}$ ) and higher concentrations of BSA provided hydrophobic coatings with a WCA of $115^{\circ}(5 \mathrm{mg}$ $\left.\mathrm{mL}^{-1}\right)$ and $140^{\circ}\left(7 \mathrm{mg} \mathrm{mL}^{-1}\right)$ as shown in Fig. S1A-F. $\dagger$ The FESEM images of the BSA derived coating that was prepared using $7 \mathrm{mg} \mathrm{mL}^{-1}$ concentration of BSA revealed the lack of an appropriate topography that is essential to display superhydrophobicity as shown in Fig. S1H. $\dagger$ Next, the presence of residual acrylate groups and the post-covalent modification of the chemically reactive BSA coating with ODA molecules were characterized with standard and widely recognized FTIR analysis as shown in Fig. 2 C. $^{49}$ The appearance of IR peaks at $1410 \mathrm{~cm}^{-1}$ and $1710 \mathrm{~cm}^{-1}$ and the reduction in the intensity of the IR peak at $1410 \mathrm{~cm}^{-1}$ with respect to the IR peak at 1710 $\mathrm{cm}^{-1}$ upon treatment with ODA unambiguously suggested the existence of residual acrylate functionalities in the BSA
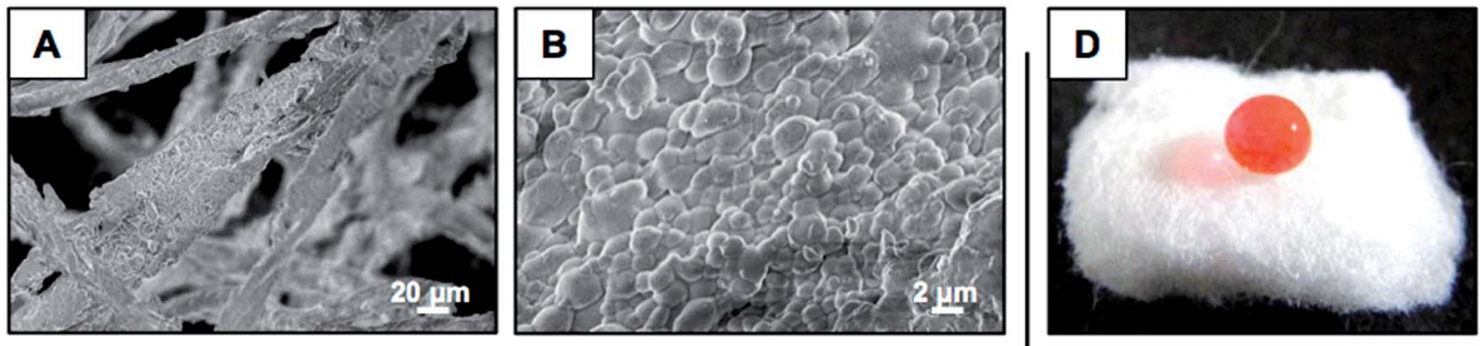

C
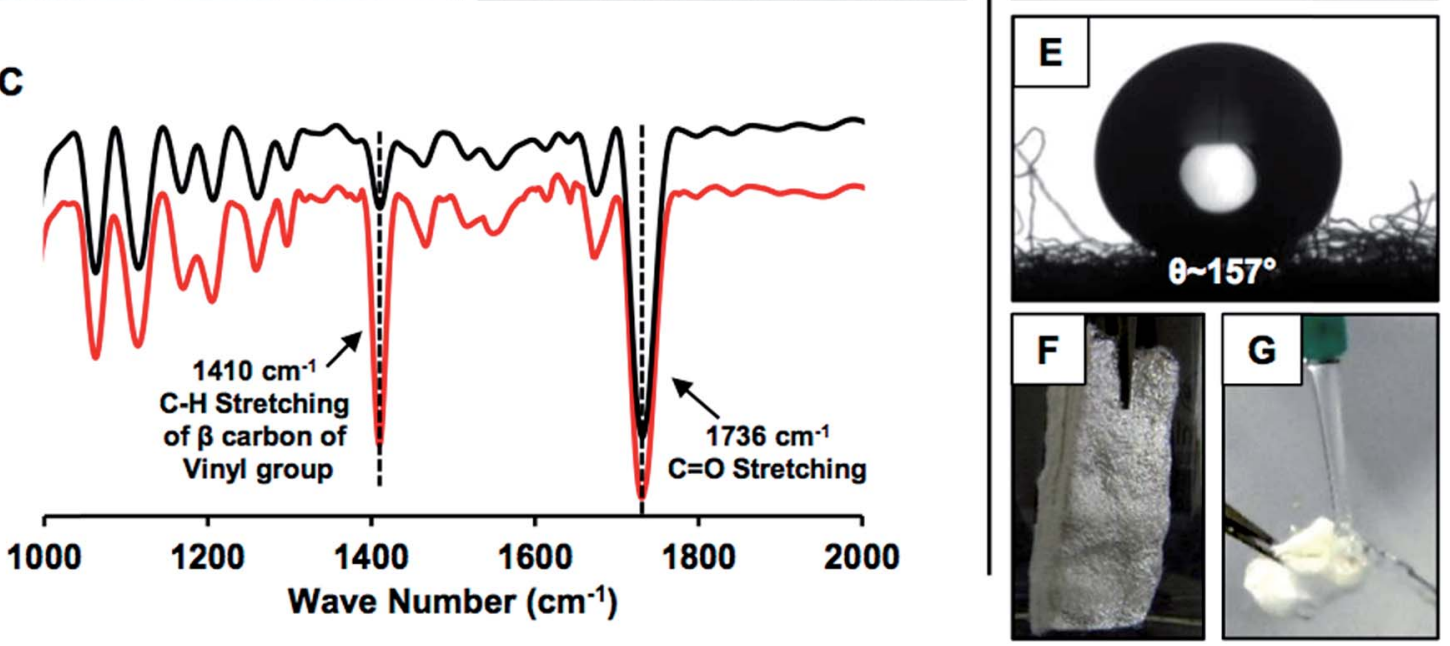

Fig. 2 (A and B) FESEM images of the BSA nanocomplex coated cotton fibres at low (A) and high (B) magnifications. (C) FTIR spectra depicting the BSA nanocomplex coated cotton before (red) and after (black) treatment with octadecylamine. (D and E) Digital image (D) and static water contact angle (E) of a beaded water droplet on the BSA protein derived superhydrophobic cotton. (F) Digital image showing a shiny interface when the superhydrophobic cotton was submerged under water, and the shiny interface revealed the existence of metastable trapped air. (G) Digital image illustrating the bouncing of a stream of water from the superhydrophobic cotton. 
coating and the mutual covalent reaction between the primary amine groups of ODA and the residual acrylate groups of the BSA coating respectively. The water wettability was examined on this synthesized material wherein a dyed (aids visual inspection) aqueous droplet (15 $\mu \mathrm{l})$ was extremely repelled by this ODA treated BSA coating with a static contact angle of $\sim 157^{\circ}$ as shown in Fig. $2 \mathrm{D}$ and E. The shiny interface (Fig. 2F) of the synthesized material underwater suggested the presence of an external phase, i.e., metastable trapped air, which contributed to its extreme heterogeneous water wettability. A stream of aqueous phase immediately bounced away upon touching the BSA protein derived superhydrophobic cotton as shown in Fig. $2 \mathrm{G}$ and Movie $1 . \dagger$ This simple demonstration revealed the existence of nonadhesive superhydrophobicity.

\section{Physical and chemical durability of the synthesized superhydrophobic interface}

Various practically relevant standard physical and chemical challenges were adopted for investigating the durability of the embedded extreme water repellency in the BSA protein derived superhydrophobic material in severe scenarios. First, different physical manipulations, i.e., bending, creasing, twisting and winding, were performed on this nature-inspired protein derived fibrous substrate and it was found that the extremely water repellent property remained intact with a static contact angle of $\sim 156^{\circ}$ and a contact angle hysteresis of $\sim 7^{\circ}$ as shown in Fig. 3A-F and S2. $\dagger$ Furthermore, severe physical abrasion tests, i.e., adhesive tape test, sand paper abrasion and finger wiping tests, were also carried out on the synthesized superhydrophobic material; however, the anti-wetting property remained intact with a static water contact angle above $155^{\circ}$ and a contact angle hysteresis of $\sim 8^{\circ}$ as shown in Fig. 3G-L and S3. $\dagger$ The FESEM images acquired after performing the adhesive tape test and sand paper abrasion revealed the presence of essential hierarchical topography which attributed to the intact water repellency as shown in Fig. S4A and B. $\dagger$ These demonstrations unambiguously suggested the existence of highly tolerant superhydrophobicity in the BSA protein derived coated medical cotton. Next, this superhydrophobic cotton was manually separated into four individual pieces arbitrarily and the water wettability was examined on these freshly exposed interfaces. The interiors of the synthesized material were also capable of displaying extreme water repellency with a water contact angle above $155^{\circ}$ and a contact angle hysteresis of $\sim 8^{\circ}$ as shown in Fig. S5A. $\dagger$ These simple demonstrations revalidated the uniform deposition of the BSA derived coating with essential topography and chemistry, which allowed it to exhibit nature-inspired superhydrophobicity. Next, the as-synthesized superhydrophobic cotton was physically deformed applying different compressive strains, and the impact of the severe physical deformation on the nature-inspired water wettability was examined in detail as shown in Fig. 4A. The applied compressive strain on the synthesized material was increased from $0 \%$ to $80 \%$ without compromising the embedded nonadhesive superhydrophobicity, and the red colored aqueous droplet beaded on the highly compressed superhydrophobic cotton with a water contact angle above $155^{\circ}$ and a contact angle hysteresis below $10^{\circ}$ as shown in Fig. 4A. On releasing the applied load, the material restored its initial shape and size, and its water repellency remained unperturbed with a static contact angle of $\sim 153^{\circ}$ as shown in Fig. S3A-C. $\dagger$ This BSA protein derived
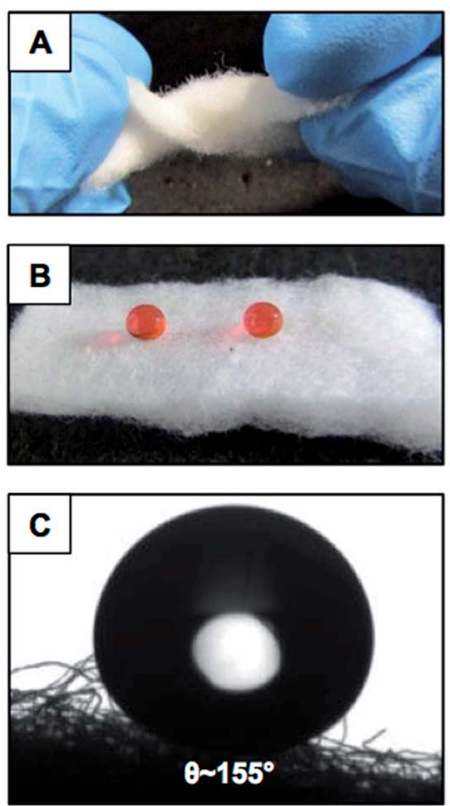
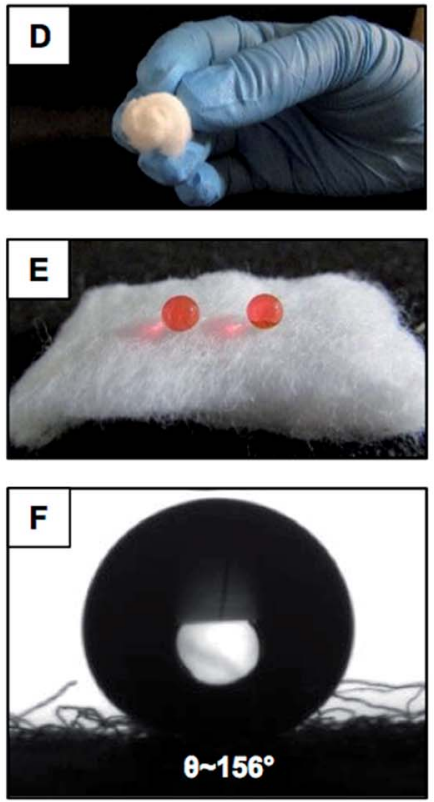
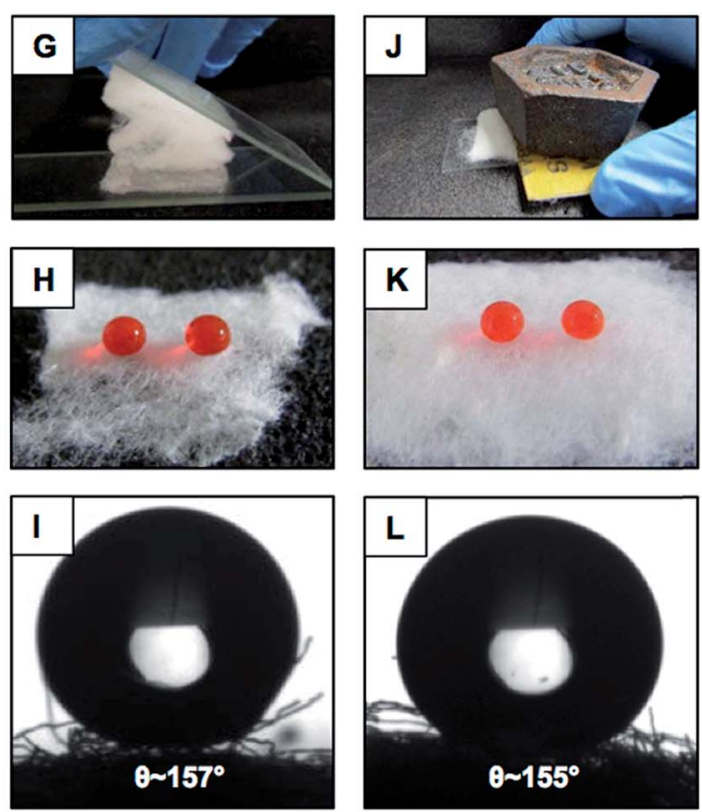

Fig. 3 Digital images ( $A, B, D$ and $E$ ) and contact angle images ( $C$ and $F$ ) depicting various physical manipulations, i.e., twisting ( $A-C)$ and winding $(\mathrm{D}-\mathrm{F})$ of the BSA derived superhydrophobic cotton. Digital images $(\mathrm{H}$ and $\mathrm{K})$ and contact angle images $(\mathrm{I}$ and $\mathrm{L})$ showing the impact of different abrasive physical durability tests-adhesive tape test (G) and sand paper abrasion test (J) - on the protein derived superhydrophobicity. 

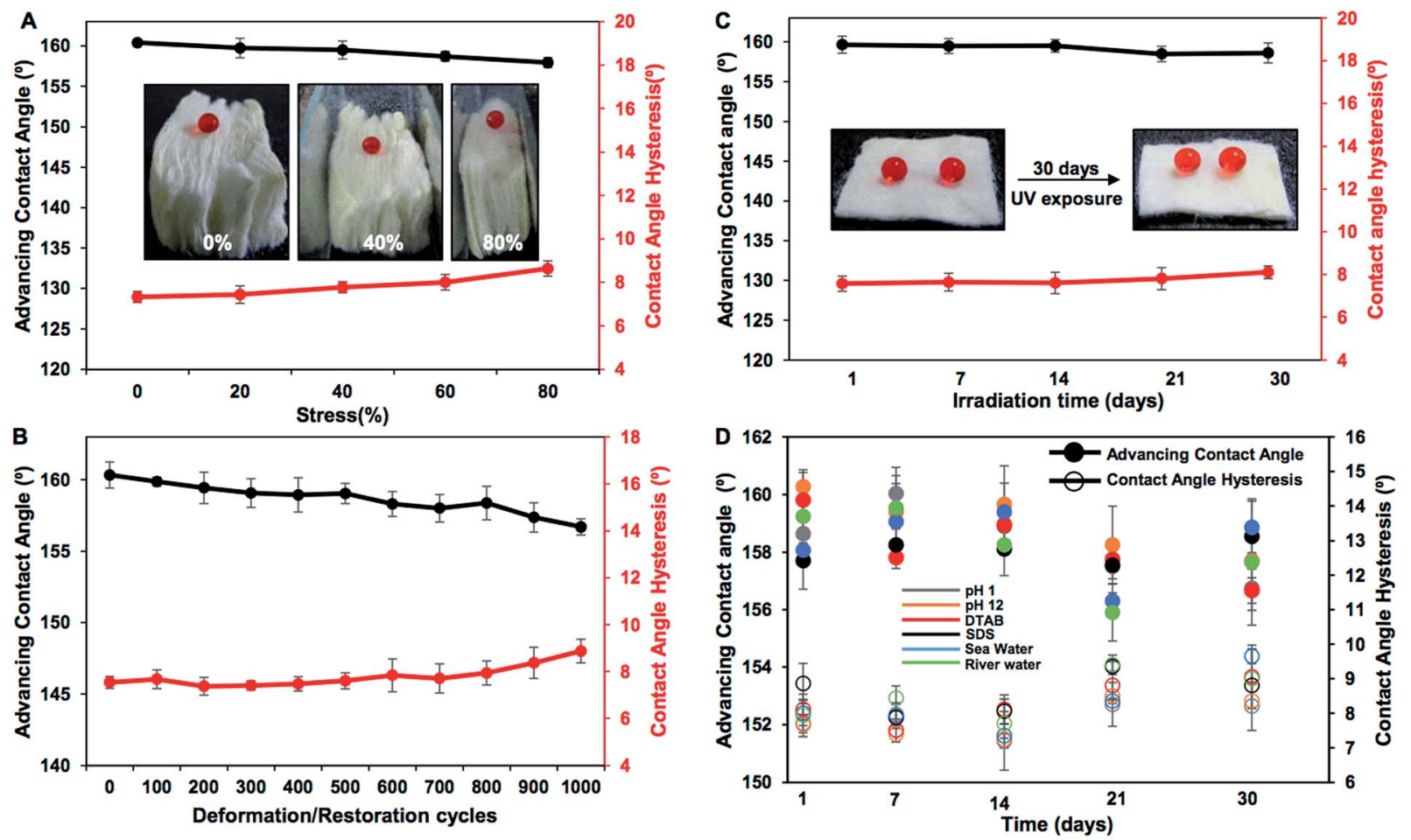

Fig. 4 (A) The plot showing the advancing contact angle (black) and the contact angle hysteresis (red) of a beaded water droplet on the superhydrophobic cotton that was manually and gradually deformed up to $80 \%$ compressive strain. The inset digital images show the superhydrophobic cotton with various compressive strains-including 0\% (left), 40\% (middle) and 80\% (right). (B) The plot illustrates the advancing contact angle (black) and contact angle hysteresis (red) of the protein coated superhydrophobic cotton after repetitively squeezing the material with $80 \%$ deformation for 1000 cycles. (C) Plot showing the advancing contact angle (black) and contact angle hysteresis (red) of the superhydrophobic cotton exposed to UV radiation for 30 days. Inset images of beaded water droplets on the substrate before and after prolonged UV irradiation. (D) Plot showing the impact of various severe chemical exposures including acidic (pH 1, grey), alkaline ( $\mathrm{pH} 12$, orange), anionic (SDS, black) and cationic (DTAB, red) surfactant contaminated water, artificial sea water (blue) and river water (green) on the extremely water repellent property of the superhydrophobic cotton for 30 days.

superhydrophobic and spongy cotton was successively squeezed with $80 \%$ compressive strain 1000 times; however, the material remained capable of displaying uninterrupted superhydrophobicity with an advancing water contact angle above $155^{\circ}$ and a contact angle hysteresis below $10^{\circ}$ as shown in Fig. 4B. This highly compressible superhydrophobic material that is capable of sustaining repetitive physical deformation is of potential interest for absorption based eco-friendly remediation of oil spills under severe practical conditions. This aspect will be discussed later in more detail. Another important durability test was performed on this synthesized material, where the superhydrophobic cotton was kept under UV radiation (at $\lambda_{\max }=254 \mathrm{~nm}$ and $365 \mathrm{~nm}$ ) for 30 days, and the water wettability was examined at regular time intervals for examining the stability of the embedded superhydrophobicity. The prolonged UV light irradiated superhydrophobic cotton continued to display extreme water repellency and the water droplets (inset of Fig. 4C) beaded with an advancing water contact angle above $158^{\circ}$ and a contact angle hysteresis below $10^{\circ}$ as shown in Fig. 4C. The superhydrophobic cotton was also exposed to various practically relevant chemically challenging conditions, i.e., extremes of $\mathrm{pH}$, artificial sea water, river water and even surfactant contaminated aqueous phases for 30 days; however, the embedded superhydrophobicity remained intact with an advancing water contact angle of above $155^{\circ}$ and a contact angle hysteresis below $10^{\circ}$ as elucidated in Fig. 4D. This unperturbed biomimicked wettability indicating the coexistence of appropriate topography and essential chemistry in the treated material.

\section{Absorption based selective removal of various oil spills under severe conditions}

These extremely water repellent interfaces were noticed to be inherently superoleophilic with an oil contact angle of $0^{\circ}$ as shown in Fig. S5 and Table S1. $\dagger$ This selective and extreme affinity (oil/oily phase) and repellency (aqueous phase) of the synthesized material towards two distinct liquids phases (oil and water) provided a facile basis to extend this durable superhydrophobic material for the eco-friendly remediation of oil/oily pollution from aqueous phases. As a proof of concept demonstration, a droplet of floating oil, i.e., motor oil at an air/water interface was selectively removed using the BSA derived superhydrophobic cotton as shown in Fig. 5A-C, S6A$\mathrm{D}$ and Movie $2, \uparrow$ where the oil phase was selectively and rapidly absorbed by the as-synthesized material, and the 

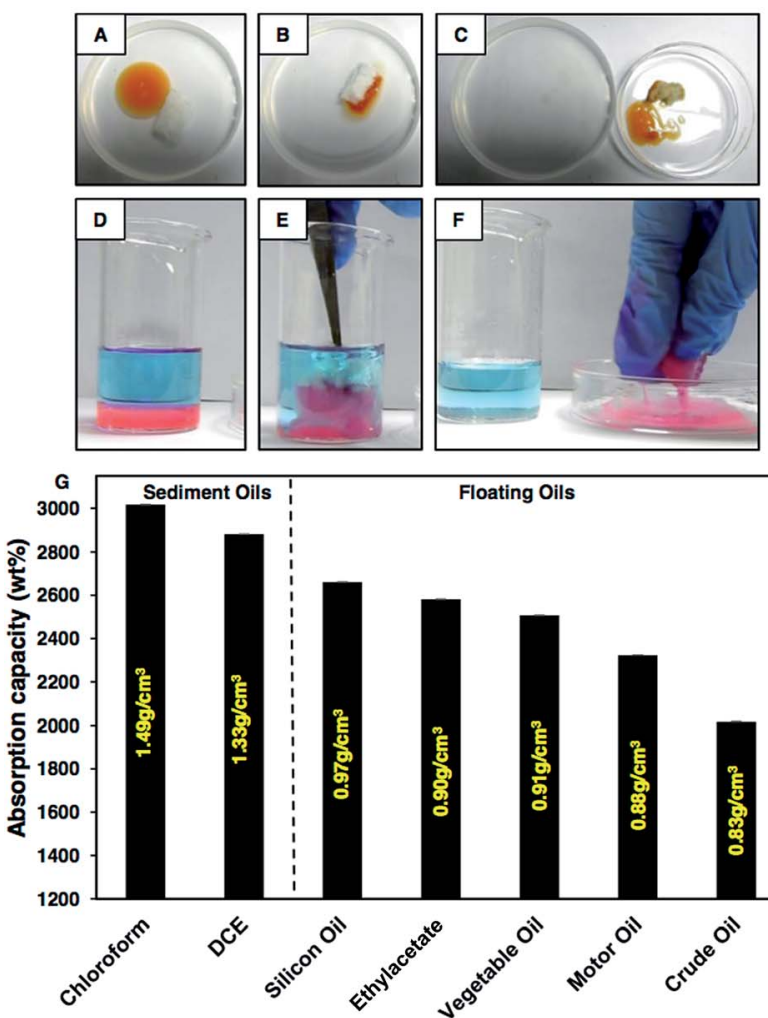

Floating Oils
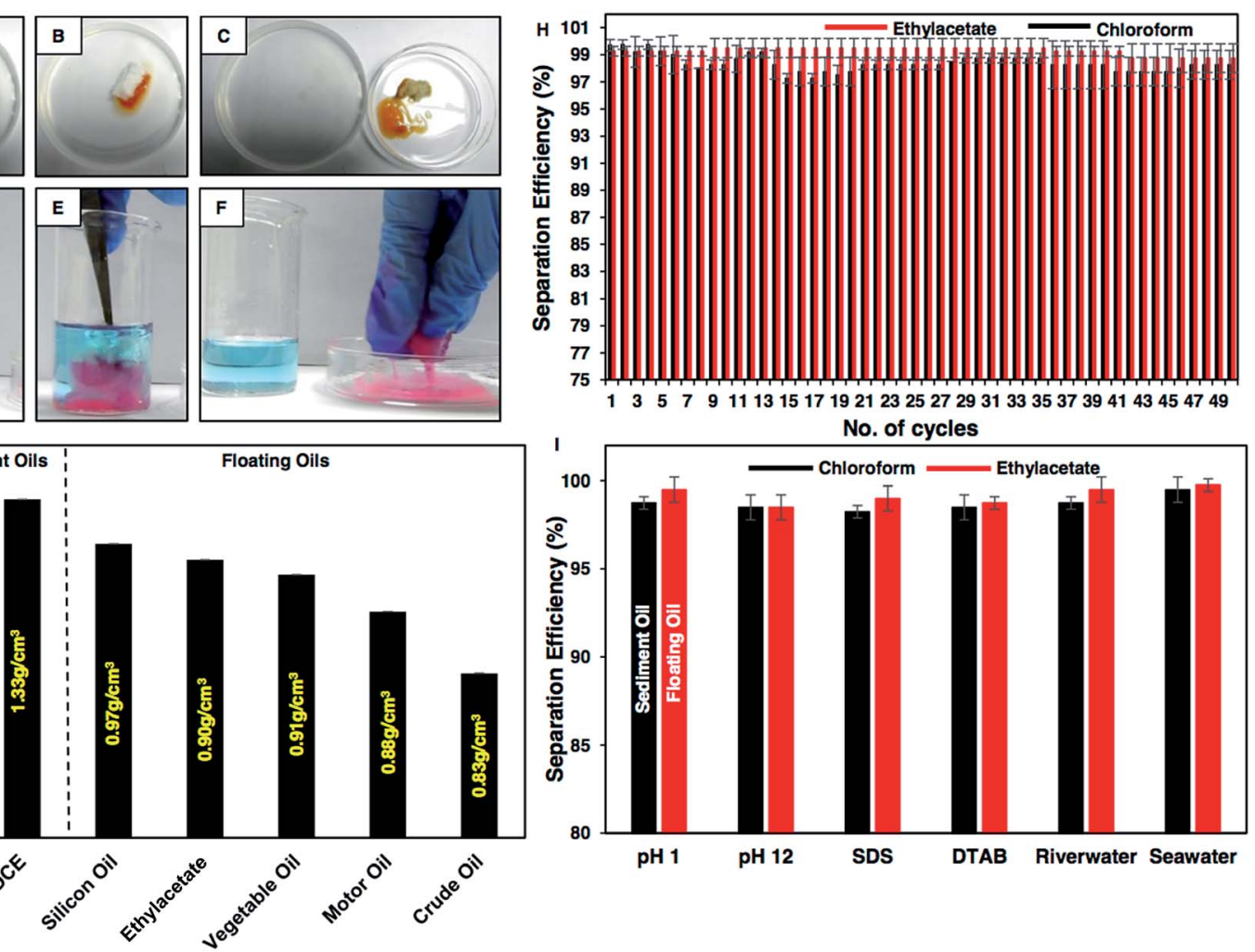

No. of cycles

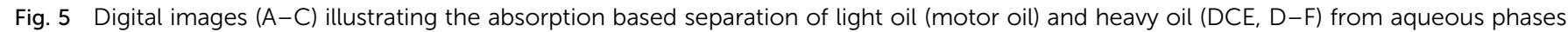

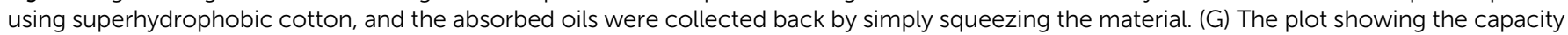

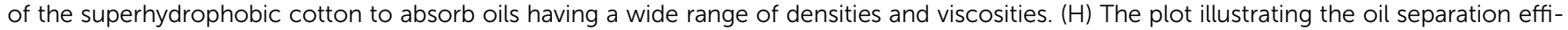

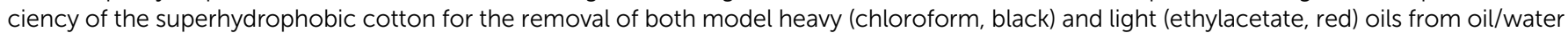

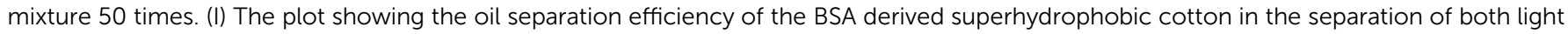

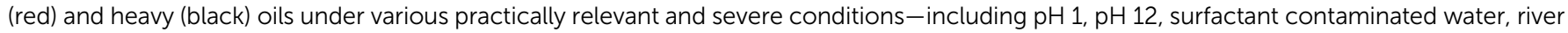
water and sea water.

absorbed oil was collected back by squeezing the superhydrophobic cotton. Furthermore, this material is capable of removing selectively sediment oil under water as shown in Fig. 5D-F, S7A-E and Movie 3. $\dagger$ The oil/water separation for silicone oil and crude oil has been illustrated in Fig. S6E-L. $\dagger$ The oil absorption capacity was found to be more than 2000 $\mathrm{wt} \%$, irrespective of the density (heavy and light oils; from $0.83 \mathrm{~g} \mathrm{~cm}^{-3}$ to $1.49 \mathrm{~g} \mathrm{~cm}^{-3}$ ) and viscosity (from $0.428 \mathrm{cP}$ to 244 cP; see Fig. S8B $\dagger$ ) of used oils (i.e. motor oil, silicone oil, ethyl acetate, crude oil, dichloroethane and chloroform) as shown in Fig. 5G. Next, the oil absorption capacity of the protein derived superhydrophobic cotton was investigated in the presence of various severe chemical conditions including extremes of $\mathrm{pH}$, sea and river water and surfactant contaminated water, where oil/aqueous mixtures were prepared using both heavy (chloroform) and light (ethylacetate) oils. The oil absorption capacity remained above $2000 \mathrm{wt} \%$, in the presence of the various severe practically relevant challenges as shown in Fig. S8A. $\dagger$ The repetitive use of the BSA derived superhydrophobic cotton for the removal of oil spillages was also examined wherein the cotton was used for the separation of oil from water followed by squeezing out the oil. The same material was reused for repetitive separation of oil/water mixtures and interestingly, the oil separation efficiency remained above $95 \%$ even after 50 cycles of subsequent reuse of the protein derived material in selective collection of either light or heavy oils as shown in Fig. $5 \mathrm{H}$. After successive $(50$ times) uses of the protein based superhydrophobic coating, it continued to display extreme water repellency with an advancing contact angle above $150^{\circ}$ and a contact angle hysteresis below $10^{\circ}$ as shown in Fig. S9. $\dagger$ The oil separation efficiency was also calculated under various harsh chemical conditions, i.e., extremes of $\mathrm{pH}$, seawater, river water and surfactant contaminated water using both heavy (chloroform) and light (ethylacetate) oils, and the oil separation efficiency remained above 95\% irrespective of the imposed chemical challenges as shown in Fig. 5I. Hence, this naturally derived superhydrophobic cotton can be used under harsh practical conditions without compromising the embedded water repellent property. The oil absorption capacity was noticed to be significantly higher compared to recently developed superhydrophobic materials.50,51 Furthermore, this BSA protein derived functional material can be strategically used for post-loading, followed by sustained release of selected bioactive drug molecules ${ }^{8}$ for various biomedical applications. 


\section{Conclusion}

To summarize, for the first time, a facile and scalable process for developing a nature-inspired water-repellent interface on an eco-friendly and naturally abundant fibrous substrate with a biodegradable protein macromolecule has been developed. The naturally synthesized bovine serum protein was rationally integrated for developing the essential hierarchical topography, and further, covalent modulation of appropriate chemistry conferred artificial superhydrophobicity. The BSA protein derived artificial superhydrophobic material was capable of sustaining repetitive (1000 times) and high (80\%) compressive strain, different physical manipulations (e.g., bending, creasing, twisting), various abrasive (adhesive tape test, abrasive sand paper abrasion etc.) physical challenges, prolonged exposure (30 days) to UV radiation and various severe chemical exposures, i.e., extremes of $\mathrm{pH}$, surfactant contaminated aqueous phases, artificial sea water, river water, etc. Moreover, the assynthesized superhydrophobic material was further exploited for selective absorption (efficiency above $2000 \mathrm{wt} \%$ ) based ecofriendly remediation of oil spills repetitively, irrespective of the density and viscosity of the contaminating oil/oily phases under various practically relevant challenges. This current approach has immense potential for developing different functional materials for various applications in practically relevant scenarios.

\section{Conflicts of interest}

There are no conflicts to declare.

\section{Acknowledgements}

We acknowledge the financial support from the Science and Engineering Research Board (YSS/2015/000818), Ministry of Electronics and Information Technology (grant no. 5(9)/2012NANO) and Department of Biotechnology (BT/PR21251/NNT/28/ 1067/2016). The Chemistry Department and the Indian Institute of Technology-Guwahati are acknowledged for their generous support. Ms Arpita Shome and Mr A. M. Rather are thankful to the institute for their PhD fellowship.

\section{References}

1 W. Barthlott and C. Neinhuis, Planta, 1997, 202, 1.

2 X. Feng and L. Jiang, Adv. Mater., 2006, 18, 3063.

3 X. M. Li, D. Reinhoudt and M. Crego-Calama, Chem. Soc. Rev., 2007, 36, 1350.

4 B. Su, Y. Tian and L. Jiang, J. Am. Chem. Soc., 2016, 138, 1727. 5 X. Zhang, F. Shi, J. Niu, Y. G. Jiang and Z. Q. Wang, J. Mater.

Chem., 2008, 18, 621.

6 W. Song, D. D. Veiga, C. A. Custódio and J. F. Mano, Adv. Mater., 2009, 21, 1830.

7 X. Yao, Y. Song and L. Jiang, Adv. Mater., 2011, 23, 719.

8 S. T. Yohe, Y. L. Colson and M. W. Grinstaff, J. Am. Chem. Soc., 2012, 134, 2016.

9 E. Ueda and P. A. Levkin, Adv. Mater., 2013, 25, 1234.
10 L. Wen, Y. Tian and L. Jiang, Angew. Chem., Int. Ed., 2015, 54, 3387.

11 J. Wang, J. A. Kaplan, Y. L. Colson and M. W. Grinstaff, Angew. Chem., Int. Ed., 2016, 55, 2796.

12 A. Gao, W. Q. Wang, D. Ha, Y. Chen and Z. P. Yang, Adv. Mater., 2016, 28, 579.

13 E. J. Falde, S. T. Yohe, Y. L. Colson and M. W. Grinstaff, Biomaterials, 2016, 104, 87.

14 C. Li, M. Boban, S. A. Snyder, S. P. R. Kobak, G. Kwon, G. Mehta and A. Tuteja, Adv. Funct. Mater., 2016, 6121.

15 C. Yu, M. Cao, Z. Dong, J. Wang, K. Li and L. Jiang, Adv. Funct. Mater., 2016, 26, 3236.

16 Y. Y. Yan, N. Gao and W. Barthlott, Adv. Colloid Interface Sci., 2011, 169, 80.

17 A. M. Rather, N. Jana, S. Begum, H. K. Srivastava and U. Manna, Green Chem., 2017, 19, 4527.

18 D. Parbat, S. Gaffar, A. M. Rather, A. Gupta and U. Manna, Chem. Sci., 2017, 8, 6542.

19 N. Kudo and Y. Kawashima, J. Toxicol. Sci., 2003, 28, 49.

20 T. L. Sun, L. Feng, X. F. Gao and L. Jiang, Acc. Chem. Res., 2005, 38, 644.

21 L. Feng, S. H. Li, Y. S. Li, H. J. Li, L. J. Zhang, J. Zhai, Y. L. Song, B. Q. Liu, L. Jiang and D. B. Zhu, Adv. Mater., 2002, 14, 1857.

22 C. H. Xue and J. Z. Ma, J. Mater. Chem. A, 2013, 1, 4146.

23 P. Roach, N. J. Shirtcliffe and M. I. Newton, Soft Matter, 2008, 4, 224.

24 L. Gao and T. J. McCarthy, Langmuir, 2006, 22, 2966.

25 M. J. Liu, S. T. Wang, Z. X. Wei, Y. L. Song and L. Jiang, Adv. Mater., 2009, 21, 665.

26 T. Verho, C. Bower, P. Andrew, S. Franssila, O. Ikkala and R. H. A. Ras, Adv. Mater., 2011, 23, 673.

27 M. Toma, G. Loget and R. M. Corn, ACS Appl. Mater. Interfaces, 2014, 6, 11110.

28 X. Mo, Y. Wu, J. Zhang, T. Hang and M. Li, Langmuir, 2015, 31, 10850.

29 Z. Han, B. Li, Z. Mu, S. Niu, J. Zhang and L. Ren, Small, 2017, 13, 1701121.

30 C. Zhou, Z. Chen, H. Yang, K. Hou, X. Zeng, Y. Zheng and J. Cheng, ACS Appl. Mater. Interfaces, 2017, 9, 9184.

31 C. R. Crick, J. A. Gibbons and I. P. Parkin, J. Mater. Chem. A, 2013, 1, 5943.

32 Z. Wang, Y. Wang and G. Liu, Angew. Chem., Int. Ed., 2016, 55, 1291.

33 G. Kwak, M. Lee and K. Yong, Langmuir, 2010, 26, 9964.

34 J. Li, C. Xu, C. Guo, H. Tian, F. Zha and L. Guo, J. Mater. Chem. A, 2018, 6, 223.

35 J. Li, L. Yan, X. Tang, H. Feng, D. Hu and F. Zha, Adv. Mater. Interfaces, 2016, 3, 1500770.

36 Y. Long, Y. Shen, H. Tian, Y. Yang, H. Feng and J. Li, J. Membr. Sci., 2018, 565, 85.

37 A. Milionis, C. S. Sharma, R. Hopf, M. Uggowitzer, I. S. Bayer and D. Poulikakos, Adv. Mater. Interfaces, 2018, 1801202.

38 N. A. Ivanovaa and A. B. Philipchenko, Appl. Surf. Sci., 2012, 263, 783.

39 H. A. Mckenzie, M. B. Smith and R. G. Wake, Nature, 1955, 176, 738. 
40 J. Xie, Y. Zheng and J. Y. Ying, J. Am. Chem. Soc., 2009, 131, 888.

41 Y. Yong, L. Zhou, Z. Gu, L. Yan, G. Tian, X. Zheng, X. Liu, X. Zhang, J. Shi, W. Cong, W. Yin and Y. Zhao, Nanoscale, 2014, 6, 10394.

42 M. Tebbe, C. Kuttner, M. Männel, A. Fery and M. Chanana, ACS Appl. Mater. Interfaces, 2015, 7, 5984.

43 A. Aires, S. M. Ocampo, D. Cabrera, L. Cueva, G. Salas, F. J. Teran and A. L. Cortajarena, J. Mater. Chem. B, 2015, 3, 6239.

44 Z. Guo, S. Zhu, Y. Yong, X. Zhang, X. Dong, J. Du, J. Xie, Q. Wang, Z. Gu and Y. Zhao, Adv. Mater., 2017, 29, 1704136.

45 L. Xie, W. Tong, Y. Dahai, J. Xu, J. Li and C. Gao, J. Mater. Chem., 2012, 22, 6053.

46 Y. Huang, Y. Luo, W. Zheng and T. Chen, ACS Appl. Mater. Interfaces, 2014, 6, 19217.
47 P.-L. Lam, S. H.-L. Kok, R. Gambari, T.-W. Kok, H.-Y. Leung, K.-L. Choi, C.-S. Wong, D. K.-P. Hau, W.-Y. Wong, K. H. Lam, Z.-X. Bian, K. K.-H. Lee and C.-H. Chui, Green Chem., 2015, 17, 1640.

48 S. L. Bechler and D. M. Lynn, Biomacromolecules, 2012, 13, 1523.

49 M. R. Weatherspoon, M. B. Dickerson, G. Wang, Y. Cai, S. Shian, S. C. Jones, S. R. Marder and K. H. Sandhage, Angew. Chem., Int. Ed., 2007, 46, 5724.

50 J. Cao, D. Wang, P. An, J. Zhang and S. Feng, J. Mater. Chem. A, 2018, 6, 18025.

51 K. Jayaramulu, K. K. R. Datta, C. Rçsler, M. Petr, M. Otyepka, R. Zboril and R. A. Fischer, Angew. Chem., Int. Ed., 2016, 55, 1178. 